\title{
Investigation on Scaled CG-Type Algorithms for Unconstrained Optimization
}

Abbas Y.AL-Bayati Khalil K. Abo Salah G. Shareef profabbasalbayati@yahoo.com

College of Computer sciences and Mathematics

University of Mosul, Iraq

Received on: 05/03/2006

Accepted on: 16/08/2006

In this paper, we describe two new algorithms which are modifications of the Hestens-stiefl CG-method. The first is the scaled CGmethod (obtained from function and gradient-values) which improves the search direction by multiplying to a scalar obtained from function value and its gradient at two successive points along the iterations. The second is the Preconditioned CG-method which uses an approximation at Hessein of the minimizing function. These algorithms are not sensitive to the line searches. Numerical experiments indicate that these new algorithms are effective and superior especially for increasing dimensionalities.

Keywords: Unconstrained Optimization, Conjugate gradient algorithm, Hestens-stiefl method, Hessein matrix.

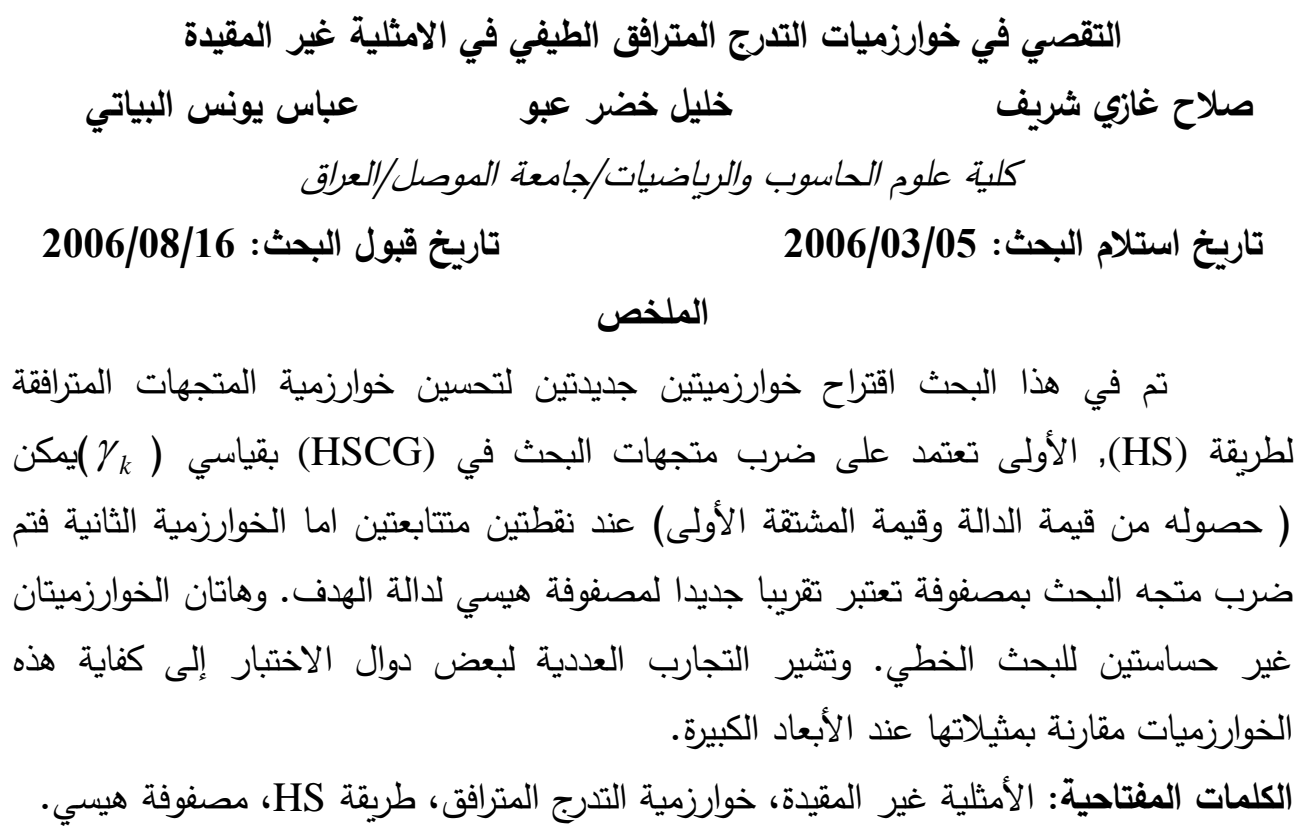

\section{Introduction:}

Unconstrained Optimization Problems expressed as: $\min f(x) ; x \in R^{n}$ 
Where $f: R^{n} \longrightarrow R$ is twice differentiable real valued function, is one of the most active areas in optimization community. virtually appearing in every human activity.

For solving these problems many efficient methods have been suggested. Excellent Presentations of these methods can be found, for example (Fletcher, 1987; Gill et-al 1981; Boyd and Vavdenberhe, 2003; Nocedal, 1992; Edwin 2001). The most useful algorithms classify in: The Conjugate gradient and its variants, Newton method and its extensions; the DFP variable metric method, many different QN methods. All these methods are iterative and consider iterations of the form:

$x_{k+1}=x_{k}+\alpha_{k} d_{k}$

where $d_{k}$ is a descent direction i.e.

$d_{k}^{T} g_{k} \prec 0$

Where $g_{k}=\nabla f\left(x_{k}\right)$ and $\alpha_{k}$ is a step length obtained by line search.Conjugate Gradient methods consider the search directions as:

$d_{k+1}=-g_{k+1}+\beta_{k} d_{k}, k \geq 1$

where the scalar $\beta_{k}$ is chosen in such a manner that the method reduces to the linear Conjugate Gradient when the function is quadratic and line search is exact. The rest of the methods define the search directions by:

$d_{k}=-G_{k}^{-1} g_{k}$,

where $G_{k}$ is a non-singular symmetric matrix. Mainly the matrix $G_{k}$ is selected as $G_{k}=I$ (identity matrix which gives the steepest descent method), $G_{k}=\nabla^{2} f\left(x_{k}\right)$ (the Newton method) or an approximation of the Hessian $\nabla^{2} f\left(x_{k}\right)$.

Different modifications are made to the CG-algorithm in different ways (see for example Hu and Story, 1990; Fletcher, 1993; Al-Baali 1985), most of these modifications are made to the search directions to improve then.

We end this general introduction by content of this paper which is organized as follows: In section(2) we review the Conjugate gradient, QNmethods and their Combinations, section(3) contains the development of the new algorithms, the last section includes the numerical results.

\section{Review of the methods:}

2-1 Conjugate Gradient Methods: 
Non-linear Conjugate gradient (CG) is one of the most useful and the earlist techniques for solving Large-scale non-linear optimization problems.

Many variants of this original scheme have been proposed, and some are widely used in practice, CG-methods only use the first order derivatives information of the objective function and need not update the Hessian matrix at each iteration. First, these are used to solve the general unconstrained optimization problems by Fletcher and Reeves (1964).

Conjugate gradient methods depend on the fact that for quadratic, if we search along a set of $\mathrm{n}$ mutually conjugate directions $d_{k}, k=1,2, \ldots, n$ (by Conjugate directions we mean that $d_{i}^{T} G d_{j}=0, \forall i \neq j$, where $\mathrm{G}$ is $n \times n$ positive matrix), then we will find the minimum in at most $n$-steps if line searches are exacts i.e.

$d_{k}^{T} g_{k+1}=0$

Moreover, if we generate this set of directions by known gradients then each direction can be simply expressed as:

$$
\left.\begin{array}{l}
d_{1}=-g_{1} \\
d_{k+1}=-g_{k+1}+\beta_{k} d_{k}, k \geq 1
\end{array}\right\}
$$

Where

$$
\beta_{k}=\frac{g_{k+1}^{T} y_{k}}{d_{k}^{T} y_{k}}
$$

And

$$
y_{k}=g_{k+1}-g_{k}
$$

$\beta_{k}$ given in equation (8) is called the Hestenes-stifel formula; clearly there are other different forms of $\beta_{k}$ such as Polak-Ribeier formula which is derived from $\beta_{k}$ in equation (8) as follows:

$$
\frac{g_{k+1}^{T} y_{k}}{d_{k}^{T} y_{k}}=\frac{g_{k+1}^{T}\left(g_{k+1}-g_{k}\right)}{d_{k}^{T}\left(g_{k+1}-g_{k}\right)}=\frac{g_{k+1}^{T} g_{k+1}-g_{k+1}^{T} g_{k}}{d_{k}^{T} g_{k+1}-d_{k}^{T} g_{k}}
$$

Assuming equation (6) and considering :

$$
d_{k}=-g_{k}+\beta_{k-1} d_{k-1}
$$

(see Edwin 2001) we get $d_{k}^{T} g_{k}=-g_{k}^{T} g_{k}$

Hence $\frac{g_{k+1}^{T} y_{k}}{d_{k}^{T} y_{k}}=\frac{g_{k+1}^{T} y_{k}}{g_{k}^{T} g_{k}}=\beta_{P R}$ 
The Fletcher Reeves formula can be derived from $\beta_{P R}$ by considering $g_{k+1}^{T} g_{k}=0$ to obtain $\beta_{F R}=\frac{g_{k+1}^{T} g_{k+1}}{g_{k}^{T} g_{k}}$

and there are many other forms. All these $\beta_{k}$ are equivalent on quadratics with exact line searches and starting with steepest descent direction, but when extended to general non-linear functions, the conjugate gradient algorithms with different $\beta_{k}$ are quite different in effacing and are considered to be not so efficient as the QN algorithms in general. The focus in this paper to the $\beta_{k}$ given in equation (8) since its original CG-method.

Finally, in many implementations of Conjugate gradient methods, The iteration (7) is restarted every $\mathrm{n}$ or $(\mathrm{n}+1)$ steps setting $\beta_{k}$ equal to zero i.e. taking steepest descent step. This ensures global convergence (Nocedal, 1992). However, different restarts are introduced (see Fletcher, 1987). One of the well-known restarts given by Powell(Powell 1977) is:

$\left|g_{k+1}^{T} g_{k}\right| \geq 0.2\left\|g_{k}\right\|^{2}$

This criterion will be used later in our suggested algorithms.

\section{2-2 Quasi-Newton Methods:}

Quasi-Newton methods are probably the most popular general purpose algorithms for unconstrained optimization problems. Many QNmethods are advantageous due to their fast convergence and absence of second order derivatives computation.

For the QN-methods assume that at the $k t h$ iteration at approximation point $x_{k}$ and $n \times n$ matrix $H_{k}$ are available. Then the methods proceed by generating a sequence of approximation points via the equation:

$$
x_{k+1}-x_{k}+\alpha_{k} d_{k}
$$

and

$$
d_{k+1}=-H_{k} g_{k}
$$

Where $H_{k}$ is an approximation of $G_{k}^{-1}$ which is corrected or updated form iteration to iteration, In general, $H_{k}$ is symmetric and positive definite, there are different choices of $H_{k}$ (see Fletcher, 1987), we list here some most popular forms (Frandsen et. al. 2004) 
$H_{k+1}^{S R}=H_{k}+\frac{\left(v_{k}-H_{k} y_{k}\right)\left(v_{k}-H_{k} y_{k}\right)^{T}}{\left(v_{k}-H_{k} y_{k}\right)^{T} y_{k}}$

is called Rank one Correction formula,

where $v_{k}=x_{k+1}-x_{k}$ and $y_{k}$ as in equation (9)

$H_{k+1}^{D F P}=H_{k}+\frac{v_{k} v_{k}^{T}}{v_{k}^{T} y_{k}}-\frac{H_{k} y_{k} y_{k}^{T} H_{k}}{y_{k}^{T} H_{k} y_{k}}$

is the DFP formula

$$
H_{k+1}^{B F G S}=H_{k}+\left[1+\frac{y_{k}^{T} H_{k} y_{k}}{v_{k}^{T} y_{k}}\right] \frac{v_{k} v_{k}^{T}}{v_{k}^{T} y_{k}}-\left[\frac{v_{k} y_{k}^{T} H_{k}+H_{k} y_{k} v_{k}^{T}}{v_{k}^{T} y_{k}}\right] \ldots \ldots \ldots \ldots \ldots
$$

All three forms satisfy the Quasi-Newton Condition

$$
y_{k}=G_{k} v_{k} \text { or } H_{k+1} y_{k}=v_{k}
$$

and maintains positive definite matrices if $H_{0}$ is positive.

We conclude our discussion of the QN methods with the following theorems:

Theorem (1): If the DFP algorithm is applied to the quadratic with Hessian $G=G^{T}$ we have

$$
H_{k+1} y_{i}=v_{i}
$$

Theorem (2): Suppose that $g_{k} \neq 0$. In the DFP algorithm, if $H_{k}$ is positive definite then so $H_{k+1}$. For proof of the theorems (1) and (2) (see Dixon, 1972)

\section{2-3 Preconditioned CG algorithm (PCG):}

The Preconditioned CG methods (PCG) first appeared in paper by Axelsson (Axelsson, 1972). It was developed with object of accelerating the convergence of the CG-method by a transformation of variables while keeping the basic properties of the method. Such transformation was introduced by Allwright (Allwright, 1972), the symmetric positive definite matrix $H$ can be factored in various ways for example as $H=L L^{T}$ where $L$ is lower triangular and non-singular (for more detail see Allwright, 1972).

Buckley (Buckley, 1978. a and b) introduced an algorithm in which conjugate gradient and quasi-Newton search directions occur together and which can be interpreted as a conjugate gradient algorithm with changing 
metric. Many authors have extended this type of algorithms (see for example Al-Bayati, 1996).

The search direction to the preconditioned (PCG) method is defined by:

$$
\begin{aligned}
& d_{1}=-H_{1} g_{1} \\
& d_{k+1}=-H g_{k}+\beta_{k} d_{k} \text { for } k \geq 1 \\
& \beta_{H S}=\frac{g_{k+1}^{T} H y_{k}}{d_{k}^{T} H y_{k}}
\end{aligned}
$$

Where $H$ is one of the forms given in equations (7) or (8) or (9). In this paper, our focus is to the $H^{P D F}$.

\section{Development of two suggested Algorithms \\ 3-1 Scaled CG-method New1 (say)}

One of the reasons for inefficiency of conjugate gradient algoriths is that non of the $\beta_{k}$ takes into consideration the effect of inexact line searches (Hu and Story, 1990). In order to do this and find an optimal $\beta$, Liu and Story (1991) introduced an algorithm that finds an optimal ( $\left.\beta_{L S}\right)$ by solving a quadratic function $f$ as:

$$
F\left(x_{k+1}, d_{k+1}\right)=\min _{\alpha} f\left(x_{k+1}+\alpha d_{k+1}\right)
$$

and

$$
\min _{\beta} F\left(x_{k+1},-g_{k+1}+\beta_{k} d_{k}\right)
$$

an then takes $d_{k+1}=\alpha^{*}\left(-g_{k+1}+\beta_{L S} d_{k}\right)$

Where $\alpha^{*}$ and $\beta_{L S}$ solve (19) and (20), respectively, the major drawback in Liu and Story CG algorithm is solving equation (20) at each iteration.

In this paper, a new form of CG-method presented with line search and so it develops conjugate search directions. This new approach will find the minimum of a $n$-dimensional quadratic function in at most $(n+1)$ function and gradient evaluations.

The motivation of the iteration

$$
x_{k+1}=x_{k}+\alpha_{k} d_{k}, \alpha_{k} \succ 0 \text {, }
$$

is that the search direction $d_{i}$ is chosen to ensure that the point $x_{i+1}$ is a minimize of the model 
$f\left(x_{k}+\alpha_{k} d_{k}\right)=f\left(x_{k}\right)+g_{k}^{T} v_{k}+\frac{1}{2} v_{k}^{T} G_{k} v_{k}$,

where $v_{k}=x_{k+1}-x_{k}=\alpha_{k} d_{k}$,

or $f\left(x_{k+1}\right)=f\left(x_{k}\right)-\alpha_{k} g_{k}^{T} g_{k}+\frac{1}{2} \alpha_{k}^{2} g_{k}^{T} \nabla^{2} f(z) g_{k}^{\prime}$,

where $z \in\left[x_{k}, x_{k+1}\right]$. If the distance between $x_{k}$ and $x_{k+1}$ is small enough we can choose $z=x_{k+1}$ and consider $\gamma\left(x_{k+1}\right) I$ as an approximation of the $\nabla^{2} f\left(x_{k+1}\right)$, where $\gamma\left(x_{k+1}\right) \in R$. This is an approximation of the Hessian at a point $x_{k+1}$ is computed using the local information from point $x_{k}$ therefore we can write

$\gamma\left(x_{k+1}\right)=\frac{2}{g_{k}^{T} g_{k}} \frac{1}{\alpha_{k}^{2}}\left[f_{k+1}-f_{k}+\alpha_{k} g_{k}^{T} g_{k}\right]$

where the step size $\alpha_{k}$ is satisfying line search conditions (Wolfe-Powell conditions) such as

$f\left(x_{k}+\alpha_{k} d_{k}\right) \leq f\left(x_{k}\right)+\rho \alpha_{k} g_{k}^{T} d_{k}$ and $\left|\nabla f\left(x_{k}+\alpha_{k} d_{k}\right)^{T} d_{k}\right| \leq-\sigma\left|\nabla f\left(x_{k}\right)^{T} d_{k}\right|$

where $0 \prec \rho \prec \sigma \prec 1$

Then we take $\gamma\left(x_{k+1}\right) I$ as an approximation of $G$. It is clear that if $\gamma\left(x_{k+1}\right) \succ 0$ this approximation will be positive definite hence to complete the method.

We must consider the situation when $\gamma\left(x_{k+1}\right) \prec 0$, i.e. if

$$
f\left(x_{k+1}\right)-f\left(x_{k}\right)+\alpha_{k} g_{k}^{T} g_{k} \prec 0
$$

In this case we can change the step size $\alpha_{k}$ as $\alpha_{k}+\eta_{k}$ s.t.

$f\left(x_{k+1}\right)-f\left(x_{k}\right)+\left(\alpha_{i}+\eta_{k}\right) g_{k}^{T} g_{k} \succ 0$

to get a value for $\eta_{k}$. Let us select a $\delta_{k} \succ 0$ small enough and consider

$\eta_{k}=\frac{1}{g_{k}^{T} g_{k}}\left[f\left(x_{k}\right)-f\left(x_{k+1}\right)-\alpha_{k} g_{k}^{T} g_{k}+\delta\right]$

then the new value of $\gamma\left(x_{k+1}\right)$ can be computed from

$\gamma\left(x_{k+1}\right)=\frac{2}{g_{k}^{T} g_{k}} \frac{1}{\left(\alpha_{k}+\eta_{k}\right)^{2}}\left[f\left(x_{k+1}\right)-f\left(x_{k}\right)+\left(\alpha_{k}+\eta_{k}\right) g_{k}^{T} g_{k}\right]$

then the new algorithm can be obtained by multiplying the search direction to scalar $\frac{1}{\gamma\left(x_{k+1}\right)}$ as follows: 


\section{The outlines of the Self-Scaling CG-method (new1):}

Step (1): Set initial point $x_{1}$ and scalar $\varepsilon$.

Step (2): For $k=1$ set $d_{1}=-g_{1}$

Step (3): For $k \geq 1$ compute $x_{k+1}=x_{k}+\alpha_{k} d_{k}$ where $\alpha_{k}$ is obtained by line search procedure

Step (4): If $\left\|g_{k}\right\| \leq \varepsilon$ stop. Otherwise go to $\operatorname{step}(5)$.

Step (5): Compute $v_{k}=x_{k+1}-x_{k}, y_{k}=g_{k+1}-g_{k}$.

Step (6): Compute $\gamma\left(x_{k+1}\right)$ from equations (25) or (28 and 29).

Step (7): Set the new $\beta_{k}$ to

$$
\beta_{k}=\frac{1}{\gamma_{k+1}} \frac{g_{k+1}^{T} y_{k}}{d_{k}^{T} y_{k}}
$$

Step (8): set $d_{k+1}=-\frac{1}{\gamma_{k+1}} g_{k+1}+\beta_{k} d_{k}$

Step (9): If restart satisfied (restart Powell 1977) go to step (2) else $\mathrm{k}=\mathrm{k}+1$ go to step (3)

\section{3-2 Self-Scaling PCG method (New2):}

In this section, a new PCG method for solving unconstrained optimization problems is proposed .

This new PCG algorithm considered here has an additional property of being invariant under scaling of the function or of its variables where the objective function is twice continuously differentiable and search direction is descent i.e. $g_{k}^{T} d_{k} \prec 0$ also we assume that line search is exact i.e. $g_{k}^{T} d_{k}=0$.

Let $d_{k}=-H_{k} g_{k}+\beta_{k} d_{k-1}$ where $\beta_{k}=\beta\left(y_{k} d_{k}, H_{k} g_{k+1}\right)$. where the matrix $H_{k}$ is an approximation of $G^{-1}$ the inverse of Hessian of the objective function $f(x)$.

One important feature of PCG method is the choice of $H_{k}$. The method requires $H_{k}$ to be positive definite to deduce directions.

Let $H_{k}=\gamma_{k+1} I$ as an approximation to the inverse Hessian where $I$ and identity matrix and $\gamma_{k+1}$ computed from (25), if $\gamma_{k+1} \succ 0$ and from (29) if $\gamma_{k+1} \prec 0$ and satisfy the condition

$H_{k}=\underset{\gamma}{\arg \min }\left\|H_{k} y_{k}-v_{k}\right\|_{2}$ 
Where $\|\cdot\|_{2}$ usual Euclidian norm, then the new 2 algorithm can be organized as follows:

The outlines of the Self-Scaling PCG method (new2):

$\operatorname{Step}(1)$ : Set $x_{1}, \varepsilon, H_{1}=I$.

$\operatorname{Step}(2)$ : For $k=1$ set $d_{1}=-H_{1} g_{1}$.

Step(3): Set $x_{k+1}=x_{k}+\alpha_{k} d_{k}$ for $k \geq 1$, where $\alpha_{k}$ optimal step size.

Step(4): If $\left\|g_{k}\right\| \leq \varepsilon$ stop. Otherwise

Check if restart equation (12) is satisfied then set $x_{k}=x_{1}$ go to step (2).

otherwise go to step (5).

Step(5): Compute $y_{k}=g_{k+1}-g_{k} ; v_{k}=x_{k+1}-x_{k}$ and $\gamma_{k}$ form equation (25).

Step(6): Compute $H_{k}=\gamma_{k} I$ satisfying (30) and $\beta_{k}=\frac{g_{k+1}^{T} H_{k} y_{k}}{d_{k}^{T} y_{k}}$.

$\operatorname{Step}(7)$ : Set $d_{k+1}=-H_{k} g_{k+1}+\beta_{k} d_{k}$ go to step (3).

\section{Numerical Results:}

All the algorithms described in this paper namely:

1. The standard HSCG method.

2. Preconditioned CG with (DFP and BFGS).

3. New1 and New2 proposed algorithms are coded in double precision FORTRAN 90. The complete set of results is given in Table (1) and Table (2). In our numerical comparison, the number of function evaluations NOF and number of iterations NOI are considered. The actual convergence criterion employed was $\left\|g_{k+1}\right\| \prec 1 \times 10^{-5}$ for all the algorithms. Wellknown test functions with different dimensions $\mathrm{n}$ are employed in this comparisons.

Table (1a) Comparison of HSCG and New1

\begin{tabular}{|c|c|c|c|c|c|}
\hline \multirow{2}{*}{ Test Fun. } & \multirow{2}{*}{ N } & \multicolumn{2}{|c|}{ HSCG } & \multicolumn{2}{c|}{ New1 } \\
\cline { 3 - 6 } & & NOI & NOF & NOI & NOF \\
\hline Dixon & 4 & 13 & 28 & 13 & 28 \\
\hline Powell (4) & 4 & 50 & 114 & 46 & 96 \\
\hline Rosen & 4 & 28 & 68 & 23 & 57 \\
\hline Cubic & 4 & 16 & 42 & 15 & $(36)$ \\
\hline Wood & 4 & 31 & 67 & 21 & 45 \\
\hline Dixon & 10 & 22 & 46 & 21 & 44 \\
\hline Powell (4) & 20 & 34 & 78 & 25 & 53 \\
\hline
\end{tabular}




\begin{tabular}{|c|c|c|c|c|c|}
\hline Rosen & 20 & 23 & 58 & 23 & 59 \\
\hline Cubic & 20 & 14 & 37 & 11 & 32 \\
\hline Wood & 20 & 52 & 107 & 31 & 66 \\
\hline Powell (4) & 100 & 129 & 263 & 67 & $(148)$ \\
\hline Rosen & 100 & 23 & 58 & 22 & 57 \\
\hline Cubic & 100 & 14 & 37 & 11 & 32 \\
\hline Wood & 100 & 69 & 140 & 85 & 174 \\
\hline Powell (4) & 500 & 458 & 921 & 68 & $(165)$ \\
\hline Rosen & 500 & 23 & 58 & 22 & 57 \\
\hline Cubic & 500 & 14 & 37 & 12 & 35 \\
\hline Wood & 500 & 69 & 140 & 86 & 176 \\
\hline Powell (4) & 1000 & 558 & 1121 & 70 & $(160)$ \\
\hline Rosen & 1000 & 23 & 58 & 22 & 57 \\
\hline Cubic & 1000 & 14 & 37 & 12 & 35 \\
\hline Wood & 1000 & 70 & 142 & 90 & 184 \\
\hline Total & & 1747 & 3657 & 786 & 1786 \\
\hline
\end{tabular}

(1b) percentages of improving the New1 method

\begin{tabular}{|l|l|l|}
\hline Tools & HSCG method & New1 method \\
\hline NOI & $100 \%$ & $55 \%$ \\
\hline NOF & $100 \%$ & $59 \%$ \\
\hline
\end{tabular}

Clearly there is an improvement of $45 \%$ in NOI and $41 \%$ in NOF for our new proposed algorithms.

Table (2a) Comparison of PCG methods with DFP, BFGS and New1

\begin{tabular}{|l|l|l|l|l|l|l|l|}
\hline Test Fun. & \multirow{2}{*}{ N } & \multicolumn{2}{|l|}{ PCG with DFP } & \multicolumn{2}{l|}{ PCG with BFGS } & \multicolumn{2}{l|}{ New2 } \\
\cline { 3 - 8 } & & NOI & NOF & NOI & NOF & NOI & NOF \\
\hline Powell (4) & 4 & 22 & 79 & 21 & 86 & 42 & 104 \\
\hline Cubic & 4 & 19 & 88 & 18 & 51 & 11 & 32 \\
\hline Dixon & 4 & 10 & 31 & 14 & 30 & 13 & 28 \\
\hline Wood & 4 & 54 & 149 & 37 & 109 & 21 & 45 \\
\hline Rosen & 4 & 23 & 63 & 34 & 87 & 30 & 81 \\
\hline Powell (4) & 20 & 36 & 135 & 38 & 123 & 30 & 83 \\
\hline Cubic & 20 & 37 & 93 & 18 & 51 & 11 & 32 \\
\hline Dixon & 10 & 31 & 90 & 22 & 47 & 21 & 44 \\
\hline Wood & 20 & 130 & 353 & 84 & 243 & 44 & 94 \\
\hline Rosen & 20 & 68 & 187 & 34 & 87 & 30 & 81 \\
\hline Powell (4) & 100 & 82 & 387 & 71 & 197 & 31 & 94 \\
\hline Cubic & 100 & 48 & 153 & 18 & 51 & 11 & 32 \\
\hline Wood & 100 & 243 & 861 & 251 & 774 & 85 & 147 \\
\hline
\end{tabular}




\begin{tabular}{|l|l|l|l|l|l|l|l|}
\hline Rosen & 100 & 155 & 389 & 34 & 87 & 30 & 81 \\
\hline Powell (4) & 500 & 54 & 284 & 50 & 148 & 36 & 113 \\
\hline Cubic & 500 & 76 & 389 & 18 & 51 & 11 & 32 \\
\hline Wood & 500 & 288 & 891 & 200 & 801 & 86 & 176 \\
\hline Rosen & 500 & 178 & 403 & 34 & 87 & 30 & 81 \\
\hline Total & & 1554 & 5025 & 996 & 3110 & 573 & 1380 \\
\hline
\end{tabular}

(2b) percentages of improving the New-2 method

\begin{tabular}{|l|l|l|l|}
\hline Tools & PCG with PDF & PCG with PFGS & New2 method \\
\hline NOI & $100 \%$ & $65 \%$ & $39 \%$ \\
\hline NOF & $100 \%$ & $62 \%$ & $28 \%$ \\
\hline
\end{tabular}

Clearly there are improvements of both standard BFGS and New2 in a bout $35 \%-61 \%$ NOI and $38 \%-64 \%$ NOF, respectively

\section{Conclusions:}

Clearly, self-scaling techniques are very effective in unconstrained optimization algorithms. The two different approaches used in this paper proved to be very effective, especially for high dimension functions.

Clearly, our numerical results indicate that there are improvements of proposed self-scaling techniques over standard DFP and BFGS algorithms. 


\section{REFERENCES}

[1] Al-Baali. M. (1985)"Descent property and global convergence of Fletcher-Reeves Method with in exact line search", J. Inst. Maths. Applns S,121-124.

[2] Al-Bayati (1996)“A New PCG Method for Unconstrained NonLinear Optimization", Journal of Abhath Al-yarmouk, Pure sci. and eng. Vol. (5), No. (1) Pp. 71-92.

[3] Allwright, J. C. (1972)“'Improving the Conditioning of Optimal Control Problems using simple methods", In Recent Mathematical Developments in Control (ed. Bell, O. J.), Academic Press.

[4] Axelsson, O. (1974)“'On Preconditioning and Convergence acceleration in sparse matrix problems", CERN Data Handling Division Report, 74-1.

[5] Boyd, S. and Vandenberhe, L. (2003) Convex optimization, Cambridge University Press.

[6] Buckley, A. G.(1978a) "A combined Conjugate Gradient quasiNewton minimization algorithm”, Mathematical Programming 15.

[7] Buckley A. G. (1978b) "Extending the relationship between the Conjugate gradient and BFGS algorithm", Mathematical Programming 15.

[8] Dixon, L. C. W. (1972) Non-linear optimization, London: English Universities Press.

[9] Edwin, K. and Stamslaw, H. (2001)An Introduction to Optimization, John Wiley and. Sons Inc .

[10] Fletcher, R. and Revees, C. (1964) "Function Minimization by Conjugate Gradients", Computer Journal, Vol.(7).

[11] Fletcher. R. (1987) "Practical Methods of Optimization", John Wiley and Sons Ltd. Second edition.

[12] Fletcher. R. (1993) "An overview of unconstrained optimization", Numerical Analysis Report NA/ 49. 
[13] Gill, Ph. E. Murray and Wright, M. (1981) "Practical Optimization", Academic Press, London.

[14] Hu,Y.F. and Story, C. (1990) "On unconstrained Conjugate gradient optimization Method and their Interrelationship", Mathematics Report Number A129.

[15] Liu, Y. and Story, C. (1991) "Efficient generalized Conjugate Gradient algorithms", Part I, theory J. of Optimization theory and Application, $\quad$ Vol. (169), No. (1).

[16] Nielsen, H. Frandsen; P. E. Jonasson; K. and Tingleff, O. (2004) "Unconstrained Optimization", Informatics and Mathematical Modeling, Technical University of Denmark, $3^{\text {rd }}$ Edition, March .

[17] Nocedal, J. (1992) "Theory of Algorithms for Unconstrained Optimization", Acta Numerica.

[18] Powell, M. J. D. (1977) "Restart procedure for the Conjugate Gradient method", Math. Programming 12. 